\title{
REVISÃO SISTEMÁTICA DAS ESTRATÉGIAS METODOLÓGICAS UTILIZADAS PARA ADAPTAÇÃO DA PRÁTICA DE ESPORTES DE AVENTURA E DA NATUREZA PARA PESSOAS COM DEFICIÊNCIA ${ }^{12}$
}

\author{
Recebido em: $12 / 12 / 2019$ \\ Aprovado em: 25/05/2020 \\ Licença:@ @ $\Theta$
}

\author{
Gabriely Steffany Paula ${ }^{3}$ \\ Andria Watanabe de Godoy ${ }^{4}$ \\ Giuliano Gomes de Assis Pimentel ${ }^{5}$ \\ Décio Roberto Calegari ${ }^{6}$ \\ Universidade Estadual de Maringá (UEM) \\ Maringá - PR - Brasil
}

RESUMO: A inclusão de pessoas com deficiência $(\mathrm{PcD})$ nas atividades físico-esportivas é um aspecto importante e ainda carente de soluções. Frente essa lacuna, buscamos identificar estratégias metodológicas utilizadas para adaptação de esportes de aventura e da natureza para PcD. Para tanto, fizemos uso de investigação bibliográfica da produção em língua portuguesa. No processo, selecionamos 07 trabalhos, que contemplaram atividades de aventura adaptada nos espaços de ambientes aquáticos e terrestres. A deficiência visual foi aquela com mais casos de adaptação para o esporte de aventura. Em geral as adaptações envolvem lugar, equipamento e didática, mas não excluem a participação de pessoas sem deficiência. As iniciativas de inclusão enfatizam a dimensão turística da aventura e a sociabilização entre familiares, colegas e PcD. Por fim, nosso levantamento estabelece tanto o Estado da Arte quanto as lacunas no conhecimento sobre quais modalidades e tipos de deficiência não possuem pesquisas publicadas sobre adaptação visando a inclusão.

PALAVRAS-CHAVE: Inclusão. Meio Ambiente. Atividades de Lazer. Turismo.

\section{SYSTEMATIC REVIEW OF METHODOLOGICAL STRATEGIES USED FOR ADAPTATION OF ADVENTURE AND NATURE SPORTS PRACTICE FOR DISABLED PEOPLE}

\footnotetext{
${ }^{1}$ Artigo premiado no $30^{\circ}$. Encontro Nacional de Recreação e lazer (ENAREL), realizado em Curitiba/PR em 2019.

${ }^{2}$ Agradecimentos: Esta pesquisa foi financiada pelo Programa institucional de bolsas de iniciação científica nas ações afirmativas - PIBIC/AF/IS/CNPq- Fundação Araucária - UEM.

${ }^{3}$ Curso de Educação Física, Universidade Estadual de Maringá - UEM/PR, Membro do GEL - Grupo de Estudos do Lazer (https://www.facebook.com/gel.uem).

${ }^{4}$ Curso de Educação Física, Universidade Estadual de Maringá - UEM/PR, Membro do GEL - Grupo de Estudos do Lazer

${ }^{5}$ DEF/Universidade Estadual de Maringá-PR, GEL - Grupo de Estudos do Lazer.

${ }^{6}$ DEF/Universidade Estadual de Maringá-PR, GEL - Grupo de Estudos do Lazer.
} 


\begin{abstract}
The inclusion of disabled people (DP) in sports activities is an important aspect and still needy solutions. Facing this gap, we sought to identify methodological estrategies used to adapt adventure and nature sports to DP. To do so, we used the bibliographical investigation of production in Portuguese. In the process, we selected seven works, which include adventure activities adapted to the spaces of aquatic and terrestrial environments. Visual impairment was the one with the most cases of adaptation to the adventure sport. In general, such adaptations involve place, equipment and didactics, but do not exclude the participation of people without disabilities. Inclusion initiatives emphasize the tourism dimension of adventure and socialization among family, colleagues and PwD. Finally, our survey determined both the state of the art and gaps in knowledge about what are the variables and types of disabilities that do not have research on adaptation to inclusion.
\end{abstract}

KEYWORDS: Inclusion. Environment. Leisure Activities. Tourism.

\title{
Introdução
}

Uma das manifestações de lazer que mais cresce associada à qualidade de vida é a atividade de aventura, tanto como experiência turística quanto sua faceta esportiva na natureza (COSTA; MARINHO e PASSOS, 2007). Segundo Schwartz (2006), os motivos para as pessoas optarem por tais práticas no contexto do lazer são vários. O principal é a vivência de algo novo, de emoções com risco controlado. Isso responde à necessidade de as pessoas saírem da rotina e dos padrões de estilo de vida. E, não obstante o reforço consumista, se busca o encontro consigo, seja porque a atividade de aventura facilita a contemplação em um ambiente natural ou porque o autoconhecimento é evocado no enfrentamento dos riscos (PIMENTEL e SAITO, 2010).

Partindo do pressuposto de que a fruição de práticas de aventura também traria benefícios emocionais às pessoas com deficiência $(\mathrm{PcD})$, nos questionamos a respeito da inclusão desse público. Ao tratarmos de deficiência, trabalhamos com o conceito presente no decreto n. 3298/99 que regulamenta a lei 7853/89 I. Assim, a deficiência compreende toda perda ou anormalidade de uma estrutura ou função psicológica, fisiológica ou anatômica que gere incapacidade para o desempenho de atividade, considerado normal para o ser humano. Essa limitação não significa impedimento, sendo importante o 
desenvolvimento de adaptações que tornem possível a participação do deficiente em práticas voltadas para sua inserção na sociedade, inclusive no lazer.

Ao observar a atuação profissional junto a $\mathrm{PcD}$, um problema é que a inclusão de pessoas com deficiência (PcD) nas atividades físico-esportivas, embora seja importante, se mostra ainda carente na formação dos profissionais do lazer (BLASCOVI-ASSIS, 1997). Essa lacuna se potencializa à medida em que as representações sociais sobre o tipo ideal do praticante de aventuras reforçam as barreiras de gênero, renda, idade e normalidade (PIMENTEL e SAITO, 2010).

Portanto, pensar a inclusão de PcD para a vivência de atividades de aventura é um desafio complementar, tanto pela especificidade da deficiência, quanto pelos preconceitos. Assim, as soluções produzidas para esse segmento precisam ser conhecidas e difundidas. Nos Estados Unidos, por exemplo, o enfrentamento dessa problemática se deu a partir de programas e de associações, sendo o canoísmo em lagos a primeira experiência (SUGERMAN, 1996). No Brasil, Auricchio (2017) destaca o protagonismo de Socorro-SP no atendimento a PcD no tocante a atividades de aventura (cascading, arvorismo, tirolesa, trilhas e rafting) como lazer turístico e físico-esportivo. Outro caso é a parceria entre universidade e associações para atendimento a PcD mental para canoísmo no mar (MORAIS; MARTINES; PIMENTEL, 2019). Portanto, ações exemplares, embora isoladas, podem iluminar a formação e atuação dos profissionais do lazer para processos inclusivos na aventura.

Todavia, não existe síntese disponível sobre a participação das PcD nesse tema. Tal saber se justifica com vistas à comunidade profissional acessar o conhecimento acumulado sobre as práticas das quais já se domina o procedimento de adaptação para a inclusão da PcD nas atividades de aventura. Considerando essa lacuna nos voltamos à 
produção acadêmica original disponível com a seguinte questão: - Quais são as adaptações necessárias para incluir as PcD nas atividades de aventura e/ou na natureza?

A partir disto apresentamos como nosso objetivo geral sistematizar o estado da arte sobre estratégias metodológicas utilizadas para adaptação da prática de esportes de aventura e da natureza com PcD. Em termos específicos, o contexto de nossas descobertas visou: identificar em qual ambiente de esporte de aventura podemos encontrar mais atividades adaptadas; e descrever as atividades adaptadas para os esportes de aventura encontrados.

\section{Metodologia}

Neste trabalho foi utilizado um modelo de revisão sistemática integrativa, cruzando a plataforma do Google Acadêmico e o Portal CAPES, na língua portuguesa. A combinação booleana "aventura na natureza" AND "deficiência" foi a mais eficiente, obtendo-se aproximadamente 146 resultados. Foram estabelecidos critérios para análise dos artigos encontrados, coletando dos artigos as informações: ano, autor, objetivo do estudo, tipo de pesquisa/análise, amostra/projeto ou programa, modalidade de aventura e principais resultados. Por meio de uma filtragem mais aprofundada desse material, foram selecionados na triagem sete (7) trabalhos publicados entre 2004 e 2016, pois descreviam detalhadamente o processo de adaptação.

Após a conclusão deste trabalho, foi publicado o estudo de Silva, et al., (2019), o qual verificou produções relacionadas à prática de atividades físicas de aventura na natureza (AFAN) para pessoas com deficiência (PcD). Eles englobaram periódicos nacionais (Qualis A1 a B2 mais a Revista SOBAMA), entre 2006 a 2016. Os descritores utilizados foram: deficiência, aventura e natureza, tendo sido selecionados os artigos de Cantorani (2014); Venditti Jr e Araújo (2008); e Venditti Jr (2012). 
Mesmo havendo dados coincidentes, que serão tratados nas discussões, há aspectos inéditos em nossa revisão que justificam sua singularidade. Por adotar padrões próximos da revisão integrativa, nosso estudo incluiu dissertações e textos de anais de congressos que, em um cenário de escassez, podem ser importantes para orientar a intervenção profissional e a formação de profissionais para atuar com PcD.

\section{Apresentação Sintética do Referencial Teórico Utilizado para a Análise dos Dados}

Reiteramos a premissa de que o mesmo benefício que uma atividade de lazer pode proporcionar a uma pessoa sem deficiência deve ser compreendido como equivalente à qualidade de vida de uma pessoa com necessidades especiais (PIMENTEL, 2003).

Nesse sentido, nos aproximamos de Blascovi-Assis (1997) sobre papel da família e da escola em uma proposta de educação pelo e para o lazer. Complementarmente, colaboradoras e a autora trouxeram mais dados, demonstrando que as famílias de crianças com deficiência acabam adotando atividades de lazer mais caseiras, muito em função das barreiras externas (falta de dinheiro, tempo e convites), embora consigam desenvolver satisfação nas práticas criadas para se adaptarem à realidade (MESSA et al., 2005). Em suma, a adesão da PcD ao lazer encontra a barreira da adaptação para a inclusão.

Frente a isso, como há muitos tipos de aventura e de deficiência, a comunidade do lazer precisa conhecer o que já foi produzido a fim de evitar repetir o mesmo estudo. Consideramos que o material a esse respeito está registrado para além dos artigos em periódicos. Por isso, o trabalho aborda o contato com a produção acadêmica por meio de uma revisão sistemática. Segundo Ercole; Melo e Alcoforado (2014) revisão sistemática é um método que envolve uma síntese rigorosa de pesquisas relacionadas a uma questão específica, principalmente a eficácia de uma ação para a solução do problema. Parte do 
rigor metodológico desta metodologia é eliminar trabalhos que não possuam delineamento empírico satisfatório e original.

Por fim, dialogamos com Silva, et al., (2019) para estabelecer aproximações e ineditismos do nosso trabalho. A propósito, reiteramos que o sucesso do país nas competições internacionais, ou seja, no esporte de rendimento não tenha repercutido proporcionalmente na produção científica relacionada ao esporte de lazer para PcD. De fato, “embora o Brasil tenha sediado os Jogos Paralímpicos de 2016, estar entre os dez primeiros países ranqueados no cenário mundial e ter uma diversidade de áreas naturais propícias para a prática de AFAN, de acesso relativamente fácil, estes fatores não resultaram em um maior número de publicações acadêmicas.” (SILVA, et a.l, 2019, p. 290)

\section{Resultados}

Dos sete artigos selecionados pudemos averiguar que as deficiências encontradas foram deficiência visual, deficiência física e múltiplas deficiências. O Quadro um distribui os resultados conforme o ambiente natural de prática e o tipo de deficiência, não sendo encontrado trabalho de adaptação de esporte aéreo de aventura na natureza.

Quadro 01: Distribuição das Modalidades Conforme Meio e Deficiência.

\begin{tabular}{|c|c|c|c|c|c|c|}
\hline \multirow[t]{2}{*}{$\begin{array}{c}\text { DEFICIENCIA/ } \\
\text { ESPORTES } \\
\end{array}$} & \multicolumn{2}{|c|}{ VISUAL } & \multicolumn{2}{|c|}{ FÍSICA } & \multicolumn{2}{|c|}{$\begin{array}{c}\text { MÚLTIPLAS } \\
\text { DEFICIENCIAS }\end{array}$} \\
\hline & AUTOR & AFAN & AUTOR & AFAN & AUTOR & AFAN \\
\hline \multirow[t]{2}{*}{ Aquático } & $\begin{array}{l}\text { Munster } \\
(2004)\end{array}$ & $\begin{array}{c}\text { Rafting } \\
\text { Mergulho } \\
\text { sub aquático } \\
\end{array}$ & - & - & - & - \\
\hline & $\begin{array}{c}\text { Souza e } \\
\text { Chaves } \\
(2015) \\
\end{array}$ & Surfe & & & & \\
\hline \multirow[t]{2}{*}{ Terrestre } & $\begin{array}{l}\text { Munster } \\
(2004)\end{array}$ & $\begin{array}{c}\text { Escalada } \\
\text { Canyoing } \\
\text { Caving } \\
\text { Trekking } \\
\end{array}$ & $\begin{array}{l}\text { Marques et } \\
\text { al. }(2011)\end{array}$ & Escalada & \multirow[t]{2}{*}{$\begin{array}{l}\text { Barroso et } \\
\text { al (2016) }\end{array}$} & \multirow{2}{*}{$\begin{array}{c}\text { Orientação à } \\
\text { caça ao } \\
\text { tesouro }\end{array}$} \\
\hline & $\begin{array}{c}\text { Carvalho } \\
(2005)\end{array}$ & Canionismo & $\begin{array}{l}\text { Nunes et } \\
\text { al. (2008) }\end{array}$ & $\begin{array}{l}\text { Espeleo } \\
\text { turismo }\end{array}$ & & \\
\hline
\end{tabular}




\begin{tabular}{c|c|c|l|l|l|c}
\hline & $\begin{array}{c}\text { Custódio } \\
(2009)\end{array}$ & Trilha natural & & \multirow{5}{*}{} & & Zarabatana. \\
\cline { 2 - 3 } & $\begin{array}{c}\text { Nunes } \text { et al } \\
(2008)\end{array}$ & $\begin{array}{c}\text { Espeleo } \\
\text { Turismo }\end{array}$ & & & & \\
\hline
\end{tabular}

Em relação aos ambientes de práticas dos esportes de aventura, constatamos que o ambiente mais praticado se caracteriza pelo terrestre com atividades adaptadas para as deficiências visual, física e múltiplas deficiências. O ambiente aquático gerou atividades adaptadas para a deficiência visual. Não foram encontradas atividades adaptadas para os ambientes aéreo, misto e urbano. Os estudos que observaram os critérios de inclusão apresentaram os seguintes resultados e conclusões:

Munster (2004) concluiu que, com a vivência de esportes de aventura, as PcD visual tiveram o acesso a diversos lugares, interação com pessoas de comunidades diferenciadas e contato com costumes típicos. As várias pessoas e grupos sociais que deram suporte à realização da pesquisa também tiveram oportunidade de conviver com as pessoas portadoras de deficiência visual e rever seus conceitos e valores com relação a essa questão.

Souza e Chaves (2015) apontam que o surfe adaptado é possível por meio de adaptações nos materiais, na prancha sendo feito a colagem de E.V.A. para a localização das áreas de posicionamento na prancha e ainda a prancha adaptada com um skate em baixo para simular a prancha em movimento. Já nas estratégias de ensino, teve o reconhecimento dos materiais, a aprendizagem da posição de espera, os movimentos da remada e a utilização de sinais sonoros. E as adaptações no ambiente em meio terrestre de início, até a prática do surfe no mar.

Já para Carvalho (2005), os exercícios desenvolvidos podem desempenhar um papel importante também no ensino do Canyoing para pessoas videntes. Os resultados demonstraram que a metodologia empregada transformou-se em uma via de mão dupla, 
na qual descobrimos que poderíamos utilizar as mesmas atividades para solucionar dificuldades de aprendizagem com videntes.

Alves e Nazari (2009) sugerem que as Atividades de Aventura, assim como diversas atividades podem ser praticadas por pessoas com algum tipo de deficiência, desde que para isso sejam realizadas adaptações necessárias e condizentes com a deficiência em questão. Dessa forma a Atividade de Aventura, efetivamente transpõe ao praticante, momentos de felicidade, medo, ansiedade, realização pessoal e principalmente, o contato com a vida.

Nunes et al. (2008), a respeito da exploração turística de cavernas, aplicaram a ferramenta Indicadores de Acessibilidade para classificar as cavidades mais adequadas às PcD. Indicadores como locomoção, condições da trilha de acesso, acesso ao pórtico da cavidade e locomoção com as PCD (físicos, visuais e com mobilidade reduzida) permitiram identificar que uma cavidade em particular (Grutão da Beleza) era a mais indicada à $\mathrm{PcD}$.

Marques et al. (2011) procuraram a presença midiática de PcD na escalada e encontraram 17 praticantes de escalada com amputação. O perfil do grupo pesquisado, principalmente no aspecto do gênero, era predominantemente masculino. A maioria dos envolvidos no levantamento é de origem norte americana e maiores de 20 anos. Nota-se a quase predominância das deficiências adquiridas, com apenas um caso de origem congênita.

Barroso et al. (2016) concluíram que as atividades promoveram a comunicação entre todos os envolvidos, indivíduos com deficiências, técnicos da instituição, familiares e instrutores de atividades de ar livre. Os participantes mostraram-se felizes, alegres, participativos e aceitaram os desafios propostos com entusiasmo, à exceção de alguns comportamentos de medo iniciais. Foram vividos momentos de liderança, partilha, 
descoberta e criatividade induzidos pelas diversas atividades. A experiência foi considerada pelos técnicos da instituição de educação especial e pelos familiares como bastante positiva, sendo unânime que deveria repetir-se mais vezes ou ser realizada com caráter regular.

Custódio (2009) propôs tarefas para cegos (congênitos e adquiridos). Tarefa 1: apontamento de marcos e estimação de distância percorrida. Tarefa 2: Apontamento geográfico. Tarefa 3: caminho de retorno. As tarefas foram realizadas em uma trilha natural de 800 metros localizada em área de proteção ambiental, localizada no Estado de São Paulo, Brasil. Foram utilizados nesse estudo materiais tecnológicos, materiais de localização (GPS e bússola), materiais de proteção e materiais auxiliares como apitos. Os resultados obtidos nessa trilha foram que os cegos adquiridos tiveram maior precisão em 4 tarefas. E as pessoas com baixa visão congênita apontaram os marcos no caminho de retorno mais próximos da realidade, pois nessa tarefa, como não existe o estímulo auditivo com apitos, o resíduo visual é utilizado para realizar a representação mental. Já nas outras tarefas, que envolviam outras percepções, as pessoas cegas congênitas demonstraram maior acurácia. $\mathrm{O}$ estudo concluiu que não houve diferenças significativas na navegação de pessoas cegas, não importando se são congênitas ou adquiridas.

Portanto, podemos sintetizar as seguintes abstrações em relação às pesquisas na área:

- $\mathrm{O}$ acesso a prática de aventura proporciona à $\mathrm{PcD}$ possibilidades turísticas de conhecer ambientes que ela não se arriscaria sem o pretexto da atividade;

- A prática do surf com PcD auditiva é possível, desde que faça as adaptações no ambiente praticado, nos materiais utilizados e nas estratégias de ensino, tendo sucesso na prática; 
- A interação de PcD com pessoas sem deficiência proporciona a estas o conhecimento de potencialidades das $\mathrm{PcD}$, minimizando preconceitos;

- A mesma metodologia de ensino pode ser aplicada as pessoas tanto não videntes como videntes, ajudando as pessoas não videntes que possuem dificuldade de aprendizagem;

- O uso de indicadores para calcular o risco e a adequação do local de aventura à $\mathrm{PcD}$ se revela como uma prática adequada e que aumenta as chances de sucesso, como no caso do espeleoturismo;

- Qualquer atividade de aventura pode ser praticada por pessoas com algum tipo de deficiência desde que tenha adaptações para que ela o pratique;

- Outro aspecto é que os praticantes da escalada são predominantemente pessoas com deficiência adquirida, mostrando que nem sempre há o impedimento de continuar praticando atividades que já praticava antes;

- As atividades de aventura proporcionam ao individuo momentos de felicidade, medo, liderança, descoberta e criatividade trazendo uma experiência positiva.

Portanto, as principais AFAN adaptadas encontradas foram o rafting, mergulho subaquático, surfe, escalada, canyoing (canionismo), caving, trekking, canionismo, trilha natural, espeleoturismo, tirolesa, orientação à caça ao tesouro e zarabatana, porém sendo atividades somente, aquáticas e terrestres, predominante terrestre, abrangendo a maior parte das pessoas com deficiência. Apresentando a deficiência visual, sendo aquela com mais casos de adaptação para o esporte de aventura, mas não excluindo a participação de pessoas sem deficiência.

Tornando possível a maior parte dessas atividades praticadas, importando realmente, o desenvolvimento de adaptações para que torne possível a participação das 
PcD. Portanto, essa limitação não significa impedimento, e sim, adaptações voltadas para a inserção na sociedade, como principal foco, o lazer.

Esse referencial selecionado forneceu algumas soluções para a prática das AFAN por PCD. Em suma, observaram que as atividades de rafting e tirolesa foram voltadas para PCD motora, com adaptações na cadeirinha para imobilização de tronco, colete e cadeira para bote adaptado. Já no Trekking, para PCD auditiva, a adaptação na comunicação se deu por meio das Libras. Por fim, destacam as trilhas ecológicas voltadas para PCD visual em um Programa de desenvolvimento sensório-motor, com adaptação na trilha e a organização das pessoas em filas ou em duplas para a prática segura (SILVA et al., 2019).

Comparando o nosso estudo com o de Silva et al. (2019), encontramos no nosso material selecionado, uma abrangência maior de atividades tanto aquáticas quanto terrestres, e ainda atividades relacionadas as pessoas com múltiplas deficiências. Já nas atividades de rafting, tirolesa, trekking e trilhas ecológicas as adaptações encontradas pelos dois estudos são bem semelhantes.

Um dado oportuno é a possibilidade de já haver modalidades estruturalmente tão organizadas na gestão dos riscos que as adaptações não necessitariam ser desenvolvidas, como no caso do rafting e da tirolesa. (SILVA et al., 2019)

Outra informação levantada por Silva et al., (2019) foi que o trekking e as trilhas ecológicas são as AFAN mais utilizada para a prática da PcD. São práticas financeiramente acessíveis por não necessitarem de equipamentos, viabilizando os custos ao acesso das PcD. Outro ponto interessante é que a deficiência visual pode ser um ponto facilitador para algumas práticas das AFAN, por não apresentarem problemas com a altura, durante as atividades apresentadas. 
Nossos estudos em comum observaram a ausência de pesquisas voltadas as PcD intelectual, sugerindo a inclusão desse grupo em pesquisas futuras. Também há poucas experiências no ambiente escolar, que - com as novas BNCC - levarão a Educação Física a ponderar sobre inclusão dos estudantes com necessidades especiais, contribuindo para a sua formação. Enfim, há demanda por desenvolvimento de adaptações, tornando possível a prática e a participação das PcD.

\section{Conclusão}

Frente ao encontrado, os estudos apenas contemplam atividades de aventura adaptada nos espaços de ambientes aquáticos e terrestres e a deficiência visual com o maior número de adaptações para a prática de esportes de aventura na natureza. Acreditamos que o baixo número de referências encontrado além de poder ser considerado uma limitação do estudo, também pode servir de estímulo para que sejam desenvolvidas novas pesquisas que abordem esportes de aventura adaptados para pessoas com deficiências.

Em relação ao estado da arte, recomendamos investimento em estudos de adaptação de esportes de aventura na natureza para o ambiente aquático e aéreo. Também entendemos que é necessário avançar os estudos para identificar os esportes de aventura no meio urbano, como, por exemplo, Wheellchair Moto Cross (WCMX) ou os esportes de aventura em meio aéreo.

Uma crítica que podemos fazer aos estudos é que eles não aprofundam a importância das entidades esportivas, como o Comitê Paralímpico, e o poder público podem desenvolver políticas ou programas para inclusão do deficiente em atividades de aventura no meio natural. 
Apesar de já existirem iniciativas que viabilizam a acessibilidade de PCD a espaços de natureza, essas ações ainda são incipientes, porém sinalizam a construção de um segmento com potencial econômico a ser explorada, para além da perspectiva da inclusão da PCD na sociedade, que deveria ser o principal objetivo dessas iniciativas.

\section{REFERÊNCIAS}

ALVES, L. S.; NAZARI, J. Atividades de Aventura e deficiência: limites e possibilidades. Efdeportes, Buenos Aires, n. 138, p.1-6, nov. 2009. Disponível em: http://www.efdeportes.com/efd138/atividades-de-aventura-e-deficiencia.htm. Acesso em: 19 set. 2019.

AURICCHIO, J. R. Formação dos profissionais da cidade de Socorro- SP em atividades de aventura no âmbito do lazer. Licere, v. 20, n.1, p. 140-160, 2017. DOI: doi.org/10.35699/1981-3171.2017.1590.

BARROSO, M. et al. Uma 'aventura inclusiva'. Atividades de natureza e aventura para pessoas com deficiência. Revista da UIIPS, Santarém-PT, v. 4, n. 1, p. 144, fev. 2016.

BLASCOVI-ASSIS, S. M. Lazer e deficiência mental: o papel da família e da escola em uma proposta de educação pelo e para o lazer. 1997. 124f. Tese (doutorado) Universidade Estadual de Campinas, Faculdade de Educação Física, Campinas, SP. Disponível em: https://www.repositorio.unicamp.br/handle/REPOSIP/275283 . Acesso em: 01 ago. 2019.

BRASIL. Decreto n. 3298/99, de 07 de jun. de 2018. Disponível em: https://www.desenvolvimentosocial.sp.gov.br/a2sitebox/arquivos/documentos/274.pdf.

São Paulo, SP, jun. 2018.

CANTORANI, J. R. H. Lazer nas atividades de aventura na natureza e qualidade de vida para pessoas com deficiência: um estudo a partir do caso da cidade de Socorro-SP. Licere, Belo Horizonte, v.17, n.1, mar. 2014.

CARVALHO, A. J. S. Esportes na natureza: estratégias de ensino do canionismo para pessoas com deficiência visual. 2005. 192f. Dissertação (Mestrado em Educação Física) - Faculdade de Educação Física. Universidade Estadual de Campinas, Campinas, 2005.

COSTA, V. L. M.; MARINHO, A.; PASSOS, K. C. M. Esportes de aventura e esportes radicais: propondo conceitos. Motriz, Rio Claro, v. 13, n. 12, p. S188, 2007. Suplemento.

CUSTÓDIO, V. Caminhada de pessoas com deficiência visual em áreas naturais: um estudo com auxílio do GPS (sistema de posicionamento global). 2009. 109 f. Tese (Doutorado em Educação Física) - Faculdade de Educação Física. Universidade Estadual de Campinas, Campinas, 2009. 
ERCOLE, F.F.; MELO, L. S.; ALCOFORADO, C. L. G. C. Revisão integrativa versus revisão sistemática. REME, v.18, n.1, p.9-12, Jan./Mar. 2014. Disponível em: http://www.reme.org.br/sumario/89. Acesso em: 19 set. 2019.

MARQUES, I. G. et al. Escalada para amputados: um estudo exploratório. In: CBAA CONGRESSO BRASILEIRO DE ATIVIDADES DE AVENTURA "ESPORTE E TURISMO: Parceiros da Sustentabilidade nas Atividades de Aventura", 6. Anais... Pelotas/RS 1 a 3 de julho 2011. Disponível em: http://www.rc.unesp.br/ib/efisica/lel/anais_cbaa/VICBAA.pdf. Acesso em: 19 abr. 2018.

MESSA, A. A.; ARAÚJO, C.O.; FREITAS, C.S.; PENNA, E.C.G.; YASUI, E.M.; GUSSON-AGUIAR, L.; FELIPPE, M.C.G.C.; BETTINI-PEREIRA, R.A.; GARCIA, R.R.; BLASCOVI-ASSIS, S.M. Lazer Familiar: A percepção de pais de crianças com deficiência sobre esta atividade. Cadernos de Pós-graduação em Distúrbios do Desenvolvimento, v. 5, p. 12-26, 2005.

MORAES, J. S.; MARTINES, I. C.; PIMENTEL, G. G. A. Práticas corporais de aventura na natureza e deficiência: uma experiência com a canoagem na baía de Guaratuba-PR. In: MUSSI, R. F. F.; COTES M.; NUNES, F. S.(org.) Lazer e meio ambiente: pesquisa, extensão e práticas pedagógicas. Goiânia: Kelps, 2019. p. 53-69.

MUNSTER, Mey de Abreu Van. Esportes na natureza e deficiência visual: uma abordagem pedagógica. 2004. 332 p. Tese (Doutorado em Educação Física) - Faculdade de Educação Física, Universidade Estadual de Campinas, Campinas-SP, 2004.

NUNES, E.; LUZ, C. S.; ANJOS, D.; GONÇALVES, A. C.; FIGUEIREDO, L. A. V.; ZAMPAULO, R. A. Inclusão social de portadores de necessidades especiais (PNEs) e a prática do turismo em áreas naturais: avaliação de seis cavidades turísticas do estado de São Paulo. Pesquisa em Turismo e Paisagem Cárstica. Campinas, SP: SBE, v. 1, n.1, p. 77-88, 2008. Disponível em: www.cavernas.org.br/ptpc/ptpc_v1_n1_077-088.pdf.

PIMENTEL, G. G. A.; SAITO, C. F. Caracterização da demanda potencial por atividades de aventura. Motriz, Rio Claro, v. 16, n. 1 p. 152-161, 2010.

Lazer: fundamentos, estratégias e atuação profissional. Jundiaí: Fontoura, 2003.

SILVA, D. P. et al., M. Atividade física de aventura na natureza para pessoas com deficiência. Licere, v. 22, p. 268-296, 2019.

SOUZA, J. V.; CHAVES R. S. O surfe adaptado para pessoas com deficiência visual: uma "onda" de sensações. Revista Adapta, Presidente Prudente, v.11, n.1, p.13-18. Jan./Dez., 2015.

SUGERMAN, D. Diversity: including people with disabilities in outdoor adventure programs. Parks \& Recreation, 44 - 1996.

VENDITTI JÚNIOR, R., ARAÚJO, P. F. de. Trilhas ecológicas com orientação para pessoas surdas. Pensar a Prática, v. 11 n.3 p.269-280, set./dez. 2008.

VENDITTI JÚNIOR, R. O ambiente natural como agente facilitador do desenvolvimento sensorial e motor para as pessoas com deficiência visual. Revista da Sobama, Marília, v. 13, n. 2, p. 49-56, jul./dez. 2012. 


\section{Endereço dos(as) Autores(as):}

Gabriely Steffany Paula

Rua Tocantins, 176

Paiçandu - PR - 87.140-000

Endereço Eletrônico: gabysteffany@outlook.com

Andria Watanabe de Godoy

Rua Visconde de Nacar, 84

Maringá - PR - 87.014-300

Endereço Eletrônico: andriagodoy@gmail.com

Giuliano Gomes de Assis Pimentel

Campus da UEM

Av. Colombo, 5790

Maringá - PR - 87.020-900

Endereço Eletrônico: ggapimentel@uem.br

Décio Roberto Calegari

Rua Tietê, 629 - ap. 307

Maringá - PR - 87.020-210

Endereço Eletrônico: deciorc@gmail.com 